\title{
Heterogeneous Expectations in An Estimated Medium-Scale DSGE Model
}

\author{
Wei ZHAO* \\ ${ }^{1}$ School of Finance and Economics, Xi'an Jiaotong University, Xi'an, China \\ ssisi001@126.com
}

Keywords: New Keynesian model; Heterogeneous expectations; Adaptive learning.

\begin{abstract}
This paper incorporates adaptive learning and heterogeneous expectations into the traditional rational expectation medium-scale DSGE model. In our model, some agents make mistakes when forecasting future macroeconomics variables, while other agents have rational expectations, so the expectations present heterogeneity. The estimation and simulation results of our model show that: (1) Public expectations in China are dominated by the adaptive learning behaviors; (2) Heterogeneous expectation behaviors reduce the responses of inflation and output to the money supply shock. Technology shock contributes most fluctuations in inflation, and investment shock plays an important role in explaining the output fluctuations.
\end{abstract}

\section{Introduction}

The micro-founded New Keynesian model is built under the hypothesis of rational expectations and is assumed to have a representative agent structure. Although adaptive learning has become increasingly important as an alternative approach for modeling private sector expectations, most of these models still assume the sector as one representative agent who is learning about the economy (see Evans and Honkapohja (2001) and Sargent (1999) for extensive overviews). The mainstream macro research usually constructs a dynamic stochastic general equilibrium model to simulate the impact of macroeconomic policies on the economy. The model assume that the expected behavior of the economic entity is completely rational, and the economic system achieves a rational expectation equilibrium. Rational expectation equilibrium hypothesis is that people have completely rational, of all available information (including the macro and micro level) full treatment, and thus the formation of expectations about future economic conditions. However, Carroll (2003), Mankiw et al. (2003), Branch (2004) and Pfajfar (2008) recently provided empirical evidence in support of heterogeneous expectations using survey data on inflation expectations. Adam (2007), Assenza et al. (2011), and Hommes (2011) found evidence for heterogeneity in learning to forecast laboratory experiments with human subjects. Evans and Honkapohja (2003, 2006), Berardi (2007), Tuinstra and Wagener (2007), Branch and McGough (2009), and De Grauwe (2010) have recently introduced examples of models with heterogeneous expectations in macroeconomics.

Public expectations of the macroeconomic situation will directly affect individual decision-making, and micro level decisions will conversely affect the macroeconomic by aggregation. As a result, the public expectations play an important role in understanding macroeconomic fluctuations, especially macroeconomic effects of economic policies. So it is necessary to consider the process of public expectations in the construction of macro models. The primary task of this paper is to introduce the expected behavior with difference in the traditional New Keynesian model. We assume that some micro individuals use rational expectations, while others use boundedly rational expectations, and quantitatively analyze the effects of heterogeneous expected behavior on macroeconomic, especially inflation dynamics. 


\section{The Model with Heterogeneous Expectations}

\section{Final Goods Producers}

Final goods producers employ a Dixit-Stiglizt aggregator: $Y_{t}=\left(\int_{0}^{1} Y_{t}(j)^{\frac{\varepsilon_{p}-1}{\varepsilon_{p}}} d j\right)^{\frac{\varepsilon_{p}}{\varepsilon_{p}-1}}$, where $j$ is an index for immediate good producer. $\varepsilon_{p}>1$ is the elasticity of substitution between different immediate goods. The profit maximization problem of final goods producer which take the final good price $P_{t}$ and intermediate $\operatorname{good} P_{t}(j)$ as given by: $\max _{Y_{t}(j)} P_{t} Y_{t}-\int_{0}^{1} P_{t}(j) Y_{t}(j) d j$, s.t. $P_{t} \frac{\varepsilon_{p}-1}{\varepsilon_{p}}\left(\int_{0}^{1} Y_{t}(j)^{\frac{\varepsilon_{p}-1}{\varepsilon_{p}}} d j\right)^{\frac{\varepsilon_{p}}{\varepsilon_{p}-1}-1} \frac{\varepsilon_{p}}{\varepsilon_{p}-1} Y_{t}(j)^{\frac{\varepsilon_{p}-1}{\varepsilon_{p}}-1}=P_{t}(j)$. This will produce a downward sloping demand curve for variety $j$ :

$$
Y_{t}(j)=\left(\frac{P_{t}(j)}{P_{t}}\right)^{-\varepsilon_{p}} Y_{t}
$$

\section{Intermediate Goods Producer}

The intermediate goods production technology is as: $Y_{t}(j)=A_{t} \bar{K}_{t}(j)^{\alpha} N_{t}(j)^{1-\alpha}$, in which the capital service is defined as: $\bar{K}_{t}(j)=u_{t} K_{t}(j)$. Firms could not freely adjust their price at each period. So they will choose input to minimize their cost: $\min _{\bar{K}_{t}(j), N_{t}(j)} W_{t}^{p} N_{t}(j)+R_{t}^{p} \bar{K}_{t}(j)$, s.t. $A_{t} \bar{K}_{t}(j)^{\alpha} N_{t}(j)^{1-\alpha} \geq\left(\frac{P_{t}(j)}{P_{t}}\right)^{-\varepsilon_{p}} Y_{t} \cdot \quad$ The first-order conditions are:

$$
R_{t}^{p}=\psi_{t}(j) \alpha A_{t} \bar{K}_{t}(j)^{\alpha-1} N_{t}(j)^{1-\alpha}, W_{t}^{p}=\psi_{t}(j)(1-\alpha) A_{t} \bar{K}_{t}(j)^{\alpha} N_{t}(j)^{-\alpha}
$$

Following Calvo(1983), the optimal price set by the firm is decided by the optimization problem:

$$
\begin{gathered}
\max _{P_{t}(j)} E_{t} \sum_{s=0}^{\infty}(\phi \beta)^{s} \frac{u^{\prime}\left(C_{t+s}\right)}{u^{\prime}\left(C_{t}\right)}\left(\frac{P_{t}(j)}{P_{t+s}}\left(\frac{P_{t}(j)}{P_{t+s}}\right)^{-\varepsilon_{p}} Y_{t+s}-m c_{t+s}\left(\frac{P_{t}(j)}{P_{t+s}}\right)^{-\varepsilon_{p}} Y_{t+s}\right) \text {. The first-order condition is given by: } \\
P_{t}^{*} \equiv P_{t}(j)=\frac{\varepsilon_{p}}{\varepsilon_{p}-1} \frac{E_{t} \sum_{s=0}^{\infty}(\phi \beta)^{s} u^{\prime}\left(C_{t+s}\right) m c_{t+s} P_{t+s}^{\varepsilon_{p}} Y_{t+s}}{E_{t} \sum_{s=0}^{\infty}(\phi \beta)^{s} u^{\prime}\left(C_{t+s}\right) m c_{t+s} P_{t+s}^{\varepsilon_{p}-1} Y_{t+s}} \equiv \frac{\varepsilon_{p}}{\varepsilon_{p}-1} \frac{X_{1 t}}{X_{2 t}}
\end{gathered}
$$

\section{Households}

Household chooses consumption, hours worked and bounds, so as to maximize the following objective function: $U\left(C_{t}, N_{t}\right)=\ln \left(C_{t}-b C_{t-1}\right)-\psi \frac{N_{t}(l)^{1+\eta}}{1+\eta}$, s.t. $C_{t}+I_{t}+\frac{B_{t+1}}{P_{t}} \leq w_{t}(l) N_{t}(l)+R_{t} u_{t} K_{t}+\frac{\prod_{t}}{P_{t}}+T_{t}-R C_{t}+\left(1+i_{t-1}\right) \frac{B_{t}}{P_{t}}$.

There sexists two costs. One is investment adjustment cost. The form of adjustment cost of investment as follows: $K_{t+1}=z_{t}\left(1-\frac{\tau}{2}\left(\frac{I_{t}}{K_{t}}-\delta\right)^{2}\right) I_{t}+(1-\delta) K_{t}$. The other one is capital utilization cost as showed by: $R C_{t}=\frac{K_{t}}{z_{t}}\left(\chi_{1}\left(u_{t}-1\right)+\chi_{2}\left(u_{t}-1\right)^{2}\right)$. The first-order conditions about consumption, utilization rate, bond, investment and capital as follows: 


$$
\left\{\begin{array}{l}
\lambda_{t}=\frac{1}{C_{t}-b C_{t-1}}-\beta b E_{t} \frac{1}{C_{t+1}-b C_{t}} \\
\lambda_{t}=\beta E_{t} \lambda_{t+1}\left(1+i_{t}\right) \pi_{t+1}^{-1} \\
R_{t}=\frac{1}{z_{t}}\left(\chi_{1}+\chi_{2}\left(u_{t}-1\right)\right) \\
\lambda_{t}=\mu_{t} z_{t}\left(\left(1-\frac{\tau}{2}\left(\frac{I_{t}}{I_{t-1}}-1\right)^{2}\right)-\tau\left(\frac{I_{t}}{I_{t-1}}-1\right) \frac{I_{t}}{I_{t-1}}\right)+\beta E_{t} \mu_{t+1} z_{t+1} \tau\left(\frac{I_{t+1}}{I_{t}}-1\right)\left(\frac{I_{t+1}}{I_{t}}\right)^{2} \\
\mu_{t}=\beta E_{t}\left(\lambda_{t+1}\left(R_{t+1} u_{t+1}-\frac{1}{z_{t+1}}\left(\chi_{1}\left(u_{t+1}-1\right)+\chi_{2}\left(u_{t+1}-1\right)^{2}\right)\right)+\mu_{t+1}(1-\delta)\right)
\end{array}\right.
$$

\section{Intermediate Labour Unions and Labour Packers}

Households supply differentiated labor input and are index by $l \in(0,1)$. Household labor is packed into a bundled labor that is sold to firms. Since household labor is imperfectly substituable, there is a downward-sloping demand for each variety of labor, which gives the household some wage-setting power. The final labor demand is $N_{t}=\left(\int_{0}^{1} N_{t}(j)^{\frac{\varepsilon_{w}-1}{\varepsilon_{w}}} d j\right)^{\frac{\varepsilon_{w}}{\varepsilon_{w}-1}}$. Where $\varepsilon_{p}>1$ is the elasticity of substitution between differentiated labor inputs which populate the unit interval. The labor packer maximize its profit while taking the wages given by: $\max _{N_{t}(l)} W_{t}^{p}\left(\int_{0}^{1} N_{t}(j)^{\frac{\varepsilon_{w}-1}{\varepsilon_{w}}} d j\right)^{\frac{\varepsilon_{w}}{\varepsilon_{w}-1}}-\int_{0}^{1} W_{t}(l) N_{t}(l) d l$. The first order condition with labor is the downward sloping demand curve as:

$$
N_{t}(l)=\left(\frac{W_{t}(l)}{W_{t}}\right)^{-\varepsilon_{w}} N_{t}, W_{t}=\left(\int_{0}^{1} W_{t}(j)^{1-\varepsilon_{w}} d j\right)^{\frac{1}{1-\varepsilon_{w}}}
$$

We still follow the assumption in Calvo(1983) for the household's wage decision. Each period there is a fixed probability $1-\phi_{w}$ that they can adjust their wage, or there is a fixed proportion $1-\phi_{w}$ that household can optimally adjust their wage. For simplicity, we do not allow wage indexation. The household will choose the optimal wage $w_{t}^{*}\left(w_{t}(l)\right)$ to maximize their utility. The Lagrangian is: $L=E_{t} \sum_{s=0}^{\infty}\left(\beta \phi_{w}\right)^{s}\left(-\psi \frac{N_{t+s}(l)^{1+\eta}}{1+\eta}+\lambda_{t+s}\left(\frac{W_{t+s}(l)}{P_{t+s}} N_{t+s}(l)\right)\right)$, s.t. $N_{t}(l) \leq\left(\frac{W_{t}(l)}{W_{t}}\right)^{-\varepsilon_{w}} N_{t}$.

And the first-order condition is given by:

$$
\left(w_{t}^{*}\right)^{1+\varepsilon_{w} \eta}=\frac{\varepsilon_{w}}{\varepsilon_{w}-1} \frac{E_{t} \sum_{s=0}^{\infty}\left(\beta \phi_{w}\right)^{s} \psi w_{t+s}^{\varepsilon_{w}(1+\eta)} \prod_{t, t+s}^{\varepsilon_{w}(1+\eta)} N_{t+s}^{1+\eta}}{E_{t} \sum_{s=0}^{\infty}\left(\beta \phi_{w}\right)^{s} \lambda_{t+s} w_{t+s}^{\varepsilon_{w}} \prod_{t, t+s}^{\varepsilon_{w}-1} N_{t+s}^{1+\eta}}
$$

Where $\prod_{t, t+s}=\frac{P_{t+s}}{P_{t}}$ is the cumulative gross price inflation between period $t$ and $t+s$. We replace $w_{t}(l)$ with $w_{t}^{*}$ since all households who optimally adjust their wages will choose the same wage. We define $H_{1 t}=E_{t} \sum_{s=0}^{\infty}\left(\beta \phi_{w}\right)^{s} \psi w_{t+s}^{\varepsilon_{w}(1+\eta)} \prod_{t, t+s}^{\varepsilon_{w}(1+\eta)} N_{t+s}^{1+\eta}, \quad H_{2 t}=E_{t} \sum_{s=0}^{\infty}\left(\beta \phi_{w}\right)^{s} \lambda_{t+s} w_{t+s}^{\varepsilon_{w}} \prod_{t, t+s}^{\varepsilon_{w}-1} N_{t+s}^{1+\eta}$. The first-order conditions became to: $\left(w_{t}^{*}\right)^{1+\varepsilon_{w} \eta}=\frac{\varepsilon_{w}}{\varepsilon_{w}-1} \frac{H_{1 t}}{H_{2 t}}, H_{1 t}=\psi w_{t}^{\varepsilon_{w}(1+\eta)} N_{t}^{1+\eta}+\phi_{w} \beta E_{t}\left(\pi_{t+1}\right)^{\varepsilon_{w}(1+\eta)} H_{1 t+1}$, $H_{2 t}=\lambda_{t} w_{t}^{\varepsilon_{w}} N_{t}+\phi_{w} \beta E_{t}\left(\pi_{t+1}\right)^{\varepsilon_{w}-1} H_{2 t+1}$.

\section{Government}

The Government consumption is just time-variant share of output $G_{t}=w_{t}^{g} Y_{t}$, where $w_{t}^{g}=\left(1-\rho_{g}\right) w^{g}+\rho_{g} w_{t-1}^{g}+\varepsilon_{t}^{g}$. Assume that government consumption balances each period and 
financed by the lump sum taxes $G_{t}=T_{t}$. If there are capitals in the model, it is almost impossible to have closed form solution for the flexible output and hence, it is impossible to have the flexible output in Taylor rule. We close the model by setting the Taylor rule as the monetary policy as:

$$
i_{t}=\left(1-\rho_{i}\right) i+\rho_{i} i_{t-1}+\left(1-\rho_{i}\right)\left(\phi_{\pi}\left(\pi_{t}-\pi\right)+\phi_{y}\left(\log Y_{t}-\log Y_{t-1}\right)\right)+\varepsilon_{t}^{i}
$$

\section{Learning Setup}

Assume the ratio $u$ of the public have adaptive learning expectations for inflation and output gap. And the relation is as:

$$
E_{t}^{a}\left(\hat{\pi}_{t+1}\right)=E_{t-1}^{a}\left(\hat{\pi}_{t}\right)+g\left[\hat{\pi}_{t}-E_{t-1}^{a}\left(\hat{\pi}_{t}\right)\right], E_{t}^{a}\left(\hat{y}_{t+1}\right)=E_{t-1}^{a}\left(\hat{y}_{t}\right)+g\left[\hat{y}_{t}-E_{t-1}^{a}\left(\hat{y}_{t}\right)\right]
$$

In which, $g \in(0,1)$ depicts the correction of the expected error by the public.

\section{Estimation and Simulation}

\section{Estimation Results}

In this section, we apply the Bayesian method to estimate the medium-scale model during the period of 1992-2016 in China, and the estimation results are shown in Table 1.

Table 1. Model estimation results

\begin{tabular}{|c|c|c|c|c|c|c|c|c|c|c|c|c|}
\hline Parameters & $\alpha$ & $\beta$ & $\delta$ & $b$ & $\tau$ & $\eta$ & $\omega$ & $\varepsilon_{w}$ & $\varepsilon_{p}$ & $\phi_{w}$ & $\phi_{p}$ & $g$ \\
\hline Estimation results & 0.33 & 0.99 & 0.03 & 0.65 & 2.00 & 1.00 & 0.20 & 10.03 & 10.00 & 0.75 & 0.75 & 0.79 \\
\hline Parameters & $\phi_{\pi}$ & $\phi_{y}$ & $\rho_{i}$ & $\rho_{a}$ & $\rho_{z}$ & $\rho_{g}$ & $\sigma_{i}$ & $\sigma_{a}$ & $\sigma_{z}$ & $\sigma_{g}$ & $u$ & \\
\hline Estimation results & 1.50 & 0.51 & 0.76 & 0.94 & 0.60 & 0.90 & 0.01 & 0.01 & 0.01 & 0.01 & 0.80 & \\
\hline
\end{tabular}

\section{Impulse Response}

We will investigate the impulse responses to various structural shocks and the contribution of those shocks to output and inflation of Chinese economy in this subsection. Fig. 1 depicts the real output and inflation rate to one unit structural shocks.

First of all, in response to a positive government shock, the output fall immediately, and the inflation has a positive effect. An improvement of the investment (a positive shock) increases the output and inflation. After a one standard deviation positive technology shock, the output gap declines along with an increase in the inflation. Turning to the monetary policy shock, it is clear that the output gap rise and the inflation fall after the shock appeared. 

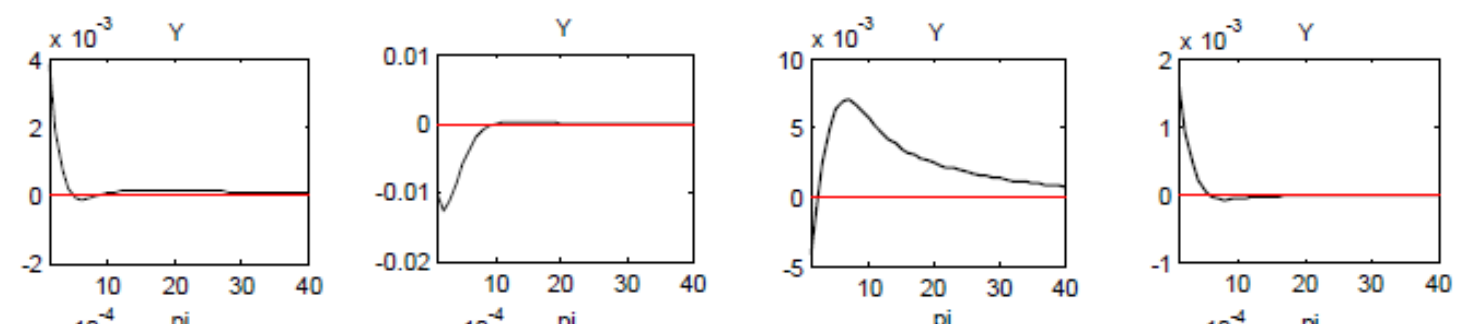

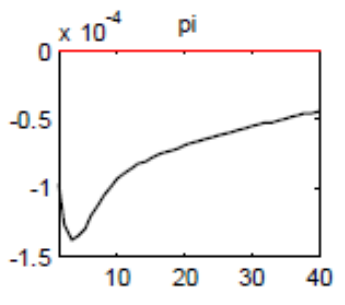

(a) Government shock

(a) Government shock

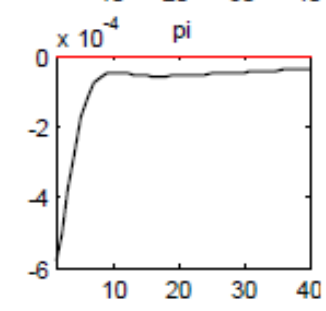

(b) Investment shock

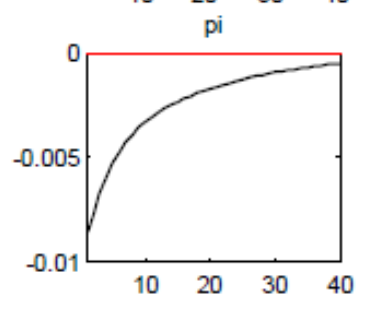

(c) Technology shock

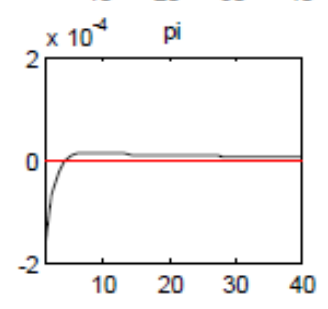

(d) Monetary policy

(b) Investment shock (c) Technology shock(d) Monetary policy

Fig 1. Impulse responses

\section{Variance Decomposition}

In order to gauge the importance of individual shocks, we compute variance decompositions in this subsection. Table. 2 presents the variance decompositions for real output and inflation. According to posterior estimates, the variance decompositions can be summarized as follows. First, technology shock contributes most fluctuations in inflation (99\%), and investment shock plays an important role to explain output swings (51\%).

Table 2. Variance decomposition (\%)

\begin{tabular}{|c|c|c|l|c|}
\hline & $\begin{array}{l}\text { Technology } \\
\text { shock }\end{array}$ & Investment shock & $\begin{array}{l}\text { Monetary policy } \\
\text { Shock }\end{array}$ & $\begin{array}{l}\text { Government } \\
\text { shock }\end{array}$ \\
\hline Inflation & 0.9969 & 0.0023 & $7.8602 \mathrm{e}-05$ & 0.0007 \\
\hline $\begin{array}{c}\text { Output } \\
\text { gap }\end{array}$ & 0.4637 & 0.5144 & 0.0035 & 0.0183 \\
\hline
\end{tabular}

\section{Summary and Concluding Remarks}

This paper has derived a general micro-founded version of the New-Keynesian model for the analysis of output and inflation dynamics in the presence of heterogeneous expectations and adaptive learning. We have modeled the individual behavior as being optimal by adaptive learning and have derived a law of motion for the output and inflation by explicitly aggregating individual decision rules. The economic model has been designed such that some agents have rational expectations while others forecast macroeconomic variables by adaptive learning. Our central findings are as follows: (1) we estimated the degree of rationality in the economy for a model with heterogeneous bounded rationality and adaptive learning. The percentage of perfectly rational agents is $80 \%$. (2)Heterogeneous expectation behaviors reduce the responses of inflation and output to the money supply shock. Technology shock contributes most fluctuations in inflation, and investment shock plays an important role to explain output swings.

\section{References}

[1] Adam, K. (2007). Experimental Evidence on the Persistence of Output and Inflation, The Economic Journal, 117(520), 603-636.

[2] Assenza, T.,P. Heemeijer, C.Hommes, D.Massaro. (2011). Individual Expectations and Aggregate Macro Behavior, CeNDEF Working Paper,University of Amsterdam. 
[3] Berardi,M. (2007). Heterogeneity and Misspecifications in Learning, Journal of Money, Credit and Banking, 31, 3203-3227.

[4] Branch, W., B. McGough. (2009). Monetary Policy in a new Keynesian Model with Heterogeneous Expectations, Journal of Economic Dynamics and Control, 33, 1036-1051.

[5] Branch, W. (2004). The Theory of Rationally Heterogeneous Expectations: Evidence from Survey Data on Inflation Expectations,” The Economic Journal, 114(497), 592-621.

[6] Calvo, G. A. (1983). Staggered Contracts in a Utility Maximizing Framework. Journal of Monetary Economics, 12, 383-398.

[7] Carroll, C. (2003). Macroeconomic expectations of households and professional forecasters, Quarterly Journal of Economics, 118, 269-298.

[8] De Grauwe, P. (2010). Animal Spirit sand Monetary Policy, Economic Theory.

[9] Evans, G.W., Honkapohja, S. (2001). Learning and Expectations in Macroeconomics, Princeton University Press, Princeton.

[10] Sargent, T.J. (1999). The Conquest of American Inflation, Princeton University Press, Princeton.

[11] Mankiw, N.,Reis, R. and Wolfer J. (2003). Disagreement about inflation expectations, NBER Macroeconomics Annual.

[12]Pfajfar, D. (2008). Heterogeneous Expectations in Macroeconomics, Phd thesis, University of Cambridge.

[13]Hommes, C.H. (2011). The Heterogeneous Expectations Hypothesis: Some Evidence from the Lab," Journal of Economic Dynamics and Control, 35, 1-24.

[14]Evans G., Honkapohja, S. (2003). Expectations and the Stability Problem for Optimal Monetary Policies, Review of Economic Studies, 70, 807-824.

[15]Evans G., Honkapohja, S.(2006). Monetary Policy, Expectations and Commitment, The Scandinavian Journal of Economics, 108, 15-38.

[16] Tuinstra,J.,Wagener F. (2007). On learning equilibria, Economic Theory, 30(3), 493-513. 